\title{
INTRATHORACIC CYSTS OF INTESTINAL AND BRONCHIAL STRUCTURE
}

\author{
BY \\ W. G. WYLLIE, M.D., F.R.C.P., \\ Physician to Out-patients, Hospital for Sick Children, Great Ormond Street \\ AND \\ ROBIN S. PILCHER, M.S. \\ Professor of Surgery, University College Hospital
}

Mediastinal cysts of intestinal and bronchial structure are rarities; one example of each is here reported, since it is only by an accumulation of records of such cases that knowledge can be gained as a basis for diagnosis and treatment, and a fatal outcome prevented. They belong to a group of mediastinal cysts of embryonic origin of which the most common and best known is the dermoid. Two characteristics mark them as distinct from the dermoids: first their posterior position in the mediastinum and secondly the early age at which they cause symptoms.

Development. At what stage of development of the embryo and by what process the various types of mediastinal cyst arise is undecided. Relative to those of entodermal origin with which this paper deals, it is known that in the period when the human embryo measures between $3 \mathrm{~mm}$. and $7 \mathrm{~mm}$. lateral grooves appear at one part of the primitive foregut demarcating a ventral respiratory and a dorsal alimentary zone. The groove areas fuse medially, separating the trachea from the oesophagus. At the caudal end of the tracheal portion the lung bud is developing, which later gives rise to paired pulmonary sacs or vesicles (Keibel and Mall, 1912). One theory holds that during this period cell buds may become separated off or sequestrated, later giving rise to the entodermal cysts under discussion. The aberrant cell groups are possibly of unspecified destiny or multipotential, since the ultimate cystic formation may be gastric, intestinal, or bronchial in structure.

Morbid anatomy and histology. The gross appearance of the cysts in previously reported cases has been varied. They may be small or large, simple or loculated, thin or thick walled. The majority have been large cysts on one side of the chest, nearly all on the right, and of these several have been much modified by multiple-stage operations before examination. Peptic ulcers have occurred in the intestinal cysts with perforation into the lung and haemoptysis. The contents of the cyst have been commented on by several authors but seldom fully examined. The fluid has been described as viscid, opalescent and milky. In
Mixter and Clifford's (1929) first case the fluid was acid, and Nicholls (1940) reported in his case excoriation of the skin round the sinus following drainage of the cyst as an empyema. In the first case reported here the fluid was not examined chemically, but at the time of operation it had a spangled appearance suggesting the presence of cholesterol crystals.

Microscopically sections of individual cysts have been reported to show in a high degree of differentiation ail the structures normally found in oesophageal, gastric, pyloric, intestinal and bronchial viscera. In a few instances two types of mucosa have been found in the same cyst, such as gastric mucosa and ciliated respiratory epithelium. The posterior mediastinal cyst recorded by Smith (1930), besides plain muscle in places in two layers showed both ciliated stratified (respiratory) epithelium and columnar epithelium with oxyntic cells (gastric). That described by Guillery (1937) was lined by epithelium both of tracheal and oesophageal types. The association of intrathoracic and abdominal cysts lined by gastric or intestinal mucosa has been recorded in several cases.

Symptomatology. Most of the cases of intestinal and bronchial cyst have occurred in infancy, and the symptoms have been mainly respiratory, but in some no respiratory disturbance was noticed by the parents. Mistaken diagnoses of pneumonia or pleural effusion have been reported in several in-

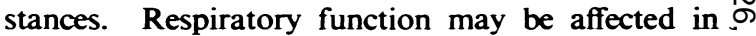
several ways. A small cyst may occlude a bronchus $N$ and cause pulmonary collapse or, if occlusion is $\mathbb{\mathrm { N }}$ partial, obstructive emphysema as in Mixter and 0 Clifford's third case, or such a cyst may compress the trachea as in the second case reported here. A $\stackrel{\bar{\Phi}}{\overparen{D}}$ large cyst will cause dyspnoea simply by its bulk $\stackrel{?}{+}$ without necessarily compressing a bronchus, and 0 this type is likely to be mistaken for pleural effusion. In some of the cases pneumonia and empyema have been diagnosed, but no clear example of true $\mathbb{\mathbb { D }}$ infection of the cyst as occurs in cysts of the lung $\bar{\sigma}$ has been found. Pneumonia is a common misdiagnosis for collapse, and the contents of a cyst $\frac{8}{0}$ might be mistaken for the pus of empyema. That 
gastric cysts may give rise to pleuritis is not surprising, as two of the cases collected by Nicholls had peptic ulcers in the wall of the cyst. In the first case reported here paracentesis yielded bloody fluid which presumably came from outside the cyst, although at operation the pleural cavity appeared clear.

Diagnosis. Without operation diagnosis cannot be made with any certainty except when aspiration of a gastric type of cyst produces acid fluid. In other cases aspiration may give no positive evidence and for small cysts may be impracticable. Radiography may show either a space-occupying lesion or its effect on the bronchus in producing collapse or obstructive emphysema. Good lateral films are essential, and unless the cyst is large will show its posterior position which distinguishes it from dermoids and cysts of pericardial origin. A large cyst may be difficult to distinguish from a pleural effusion unless (as in the first case) it has a rounded contour in the costophrenic sulcus. Bronchoscopy is of little help when the cyst is large, but in the case of small cysts the clinical picture may resemble that of foreign body or tuberculous adenitis, and here bronchoscopy is essential. The Mantoux test may also be of help if tuberculous adenitis is to be considered. Lipiodol bronchography will add no useful information unless bronchiectasis has supervened, but lipiodol injection into the cyst may be useful to demonstrate its shape and size. It seems impossible to distinguish between a large posterior mediastinal cyst and a ganglioneuroma. Erosion of bone, although suggestive of ganglioneuroma, may be caused by a cyst as in one of Mixter and Clifford's cases. In an infant of three months Guillery found at post-mortem one cyst of tracheal and oesophageal structure in the posterior mediastinum and another smaller cyst within the vertebral canal at the level of the fifth dorsal body. Symptoms of spinal compression are not mentioned, but had the child lived a dumb-bell ganglioneuroma would probably have been suspected. Paterson and Pilcher (1941) record a case of a dumb-bell ganglioneuroma in a child with a large right upper intrathoracic portion but only a small prolongation into a contiguous intervertebral foramen without pressure involvement of the cord.

\section{Case reports}

Case 1. Cyst of gastric structure. Female infant, aged twelve months, weighing $17 \mathrm{lb}$., born at full term, normal labour, with birth weight $6 \frac{1}{4} \mathrm{lb}$. From the sixth month the mother thought the infant's abdomen was unduly large, and she found the child took its feeds badly and gained little after the seventh month. There was no history of cough or infection.

At twelve months the infant was admitted to the Hospital for Sick Children, Great Ormond Street, and on examination of the abdomen the edge of the liver was felt three fingerbreadths below the costal margin; the spleen was palpable on inspiration; there was no ascites and no tenderness.
The chest was pigeon-breasted with recession of the lower intercostal spaces on inspiration; the right half of the chest was markedly dull all over, with absence of breath sounds and of râles. The heart was displaced to the left with the apex beat in the axillary line. The respiration rate was within normal limits (25 to 40 ), the temperature normal, and the general condition of the infant fair.

An x-ray showed displacement of the trachea and heart to the left, and a uniform opacity of all of the right half of the thorax, except for a small clear area in the costo-phrenic angle, bordered on the third side by the convex edge of the opaque shadow. The lateral film in this case showed less opacity in the posterior part of the chest than was expected and no outline of the tumour could be seen. A provisional diagnosis of teratoma or of ganglioneuroma was made.

By bronchoscopy (Mr. J. Crooks, October 6, 1941), under ether and chloroform, the right bronchus was difficult to find and appeared flattened, the left normal; there was no excess secretion in either.

On paracentesis 10 c.c. of blood-stained fluid was easily obtained from the right chest, and the needle on being advanced impinged against a hard mass. The fluid deposit showed numerous serosal cells and some "fat " phagocytes. Five days after aspiration the chest was screened and both sides of the diaphragm were seen to move well though the right half was at a lower level than the left: the right costo-phrenic angle was clear. On deep inspiration a rounded shadow was noticeable in the right lung field unattached to the chest wall laterally or to the diaphragm. Further $\mathrm{x}$-rays were taken for evidence of bone erosion but none was found.

October 16, 1941. Operation by R.S.P. Anaesthetic: intratracheal gas, oxygen, ether by Miss Byrde. The chest was opened by a long intercostal incision on the right side. The pleural cavity was opened anteriorly and it was found that the right side of the chest was almost entirely filled by a cystic swelling with a thick vascular wall. The collapsed right lung could be seen in front of and below the swelling. The cyst was extrapleural, what was left of the pleural cavity lying anterior to it. The cyst seemed to have its main attachment in the paravertebral gutter. To facilitate manipulation the cyst was aspirated, $8 \mathrm{oz}$. of pale yellow spangled fluid being removed. The pleura over the cyst was incised and a plane of cleavage between it and the true wall of the cyst was easily found. This plane of cleavage was extended by blunt dissection and the whole cyst was removed by an extrapleural dissection. Separation at the- lower pole and from the mediastinal structures was easy, but the cyst was more adherent in the paravertebral gutter at the apex of the chest and it was from this part that it appeared to derive its blood supply. After removal of the cyst there was left a large extrapleural cavity with a wide opening into the pleura where the latter had been incised. The pleura was stitched back to the chest wall to prevent it flapping across the chest. The chest was closed without drainage. The child stood the operation well and was transferred to an oxygen tent directly after operation. The post-operative course gave rise to no anxiety. Oxygen therapy was continued until the second morning after operation. X-ray on the second day showed the right lung beginning 

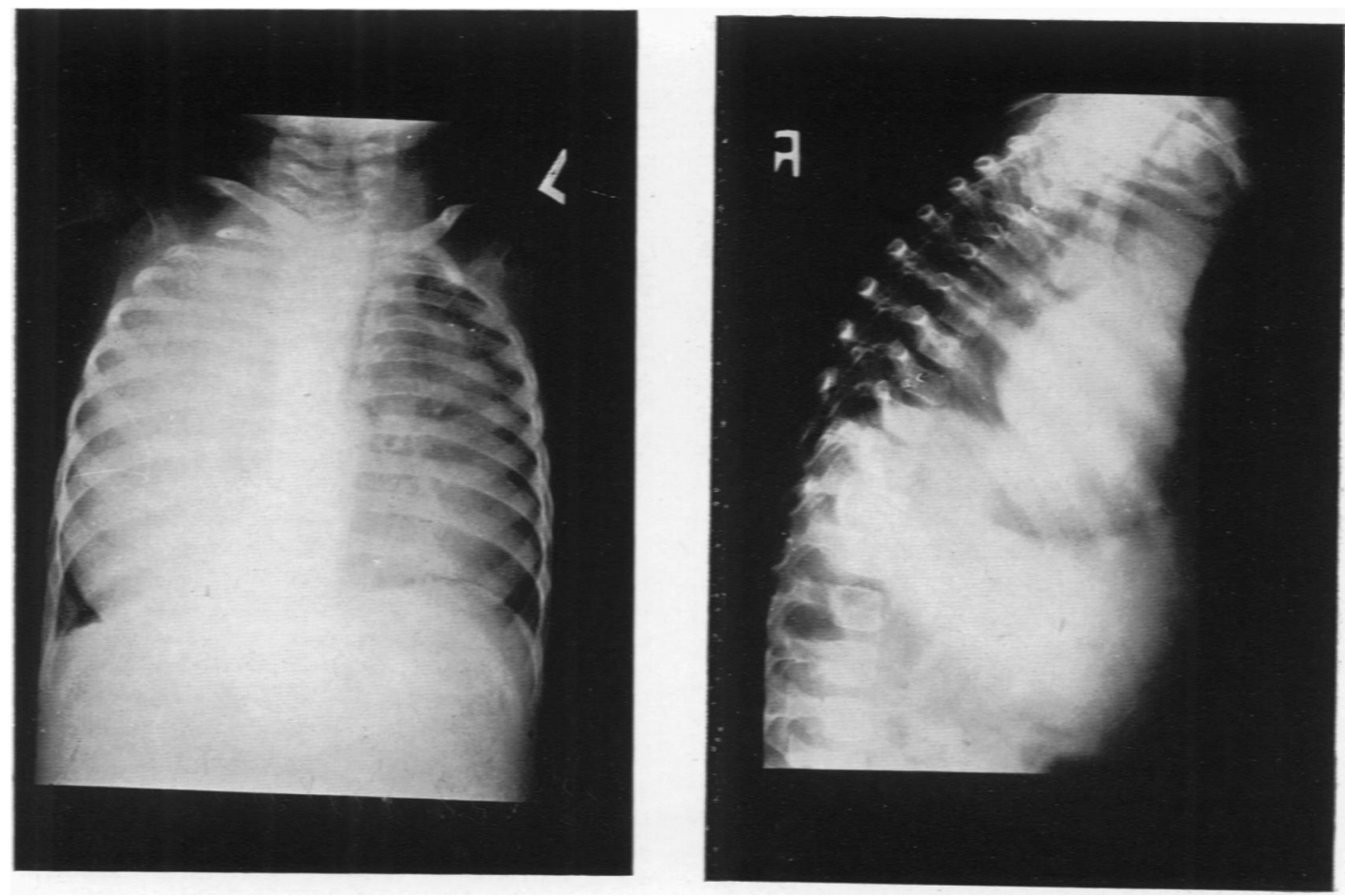

FIG. 1 and 2.-Case 1. Pre-operative $x$-rays showing cyst occupying right side of chest and displacing mediastinum to the left. The edge of the cyst can be seen outlining a small translucent area in the
costophrenic angle.
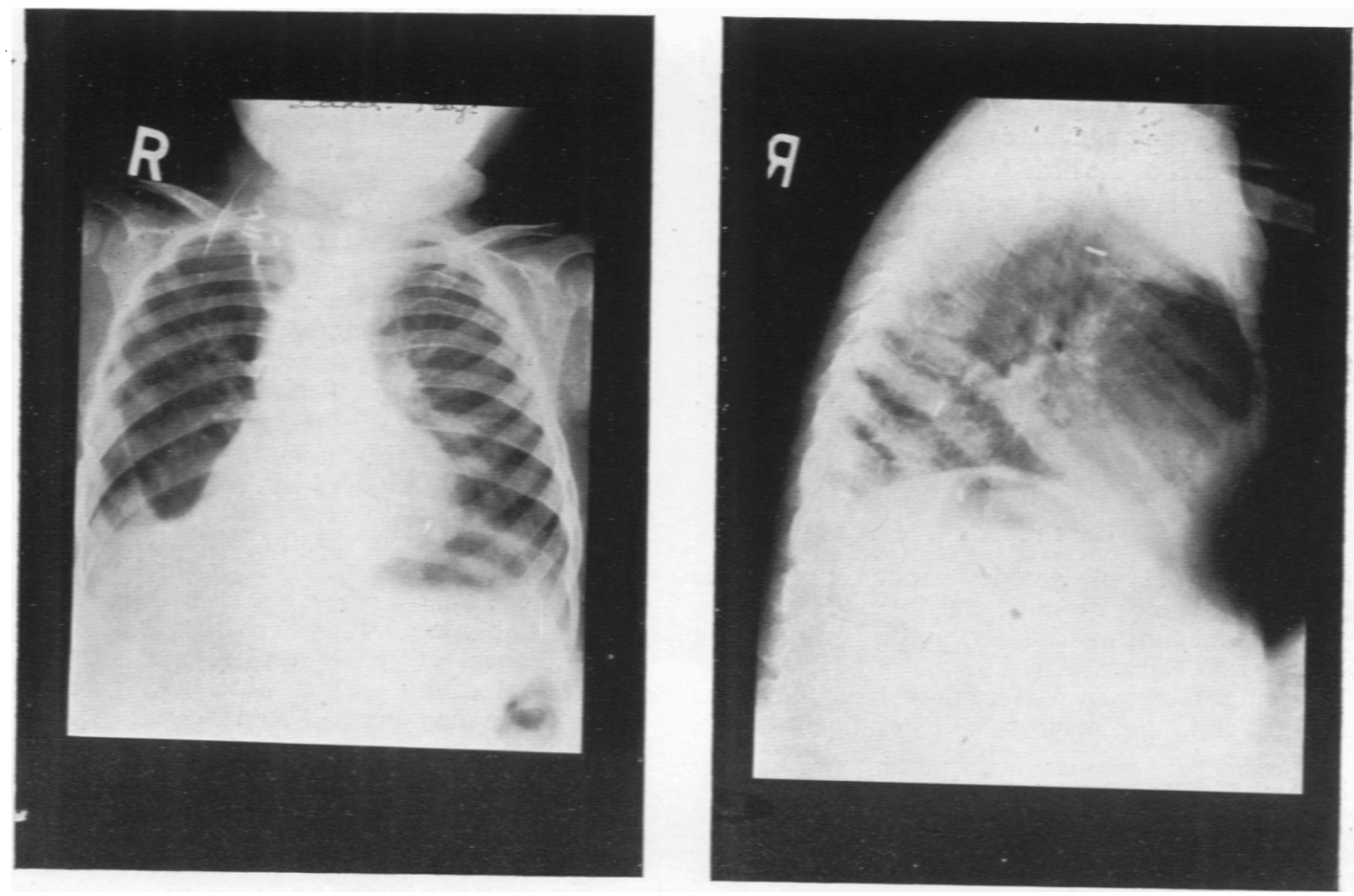

FIG. 3 and 4.-Case 1. Post-operative x-rays showing right lung expanded. 
to expand but the mediastinum still displaced to the left. There was very little effusion in the pleura. $\mathrm{X}$-ray a fortnight after operation showed that the right lung had re-expanded. After operation the child had signs of sympathetic paralysis of the right upper limb and right side of the face. This was not present before operation and no doubt the sympathetic was damaged during liberation of the upper part of the cyst.

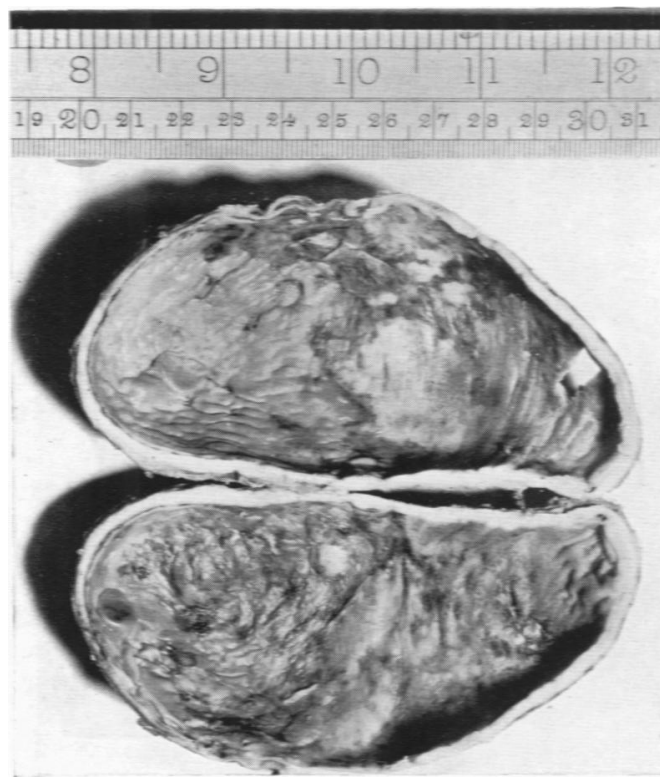

FIG. 5.-Case 1. Cyst opened longitudinally.

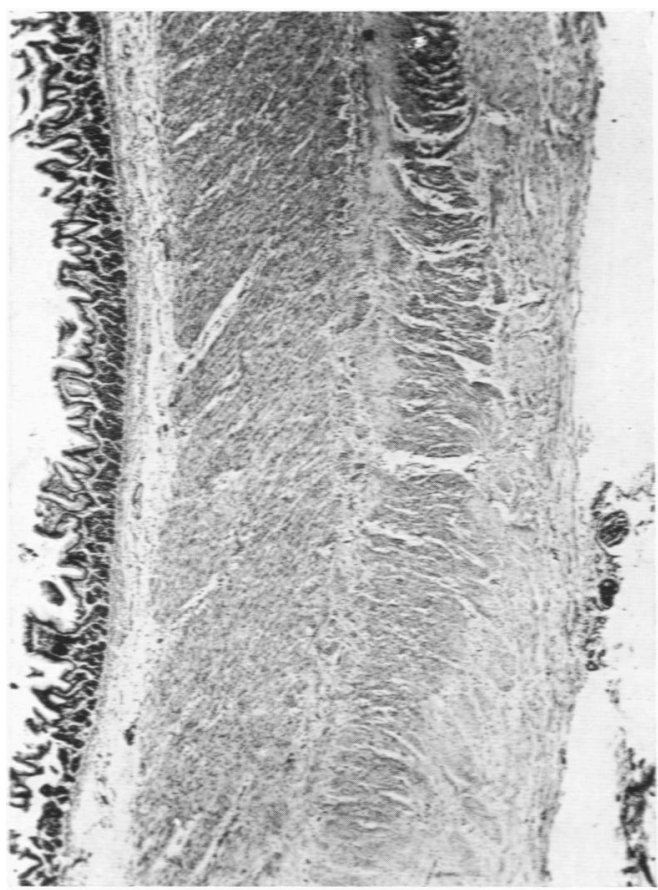

The cyst, diminished in size by aspiration, had a wall varying in thickness from 1 to $5 \mathrm{~mm}$., its outer surface was shaggy and its inner surface lined with what looked like mucous membrane. Section of the wall of the cyst closely resembled that of a normal stomach with typical gastric mucous membrane and three layers of muscle fibres.

Case 2. Cyst of bronchial structure. Female infant, aged eighteen months, weighing $27 \mathrm{lb}$., progressed satisfactorily up to the sixth month when she had a sudden attack of wheezing, accompanied by a spasmodic cough for seven days. She was free from symptoms for the next two months, when she had a similar dyspnoeic attack, since when she had not been free from wheezing and a brassy cough. For the past nine months the symptoms had not progressed. There was no family history of allergy or of tuberculosis.

At eighteen months the infant was admitted to the Hospital for Sick Children, Great Ormond Street (November 25, 1941). She was active, cheerful, but had an almost constant wheeze or stridor: respirations were not usually increased (25 to 30 ). There were frequent bouts of coughing with a brassy character, as if something were choking her, in which she became much distressed and breathed rapidly. On examination of the chest nothing abnormal was found except recession of the lower ribs and intercostal spaces. After her bouts of coughing the wheezing was more noticeable for a time and catarrhal sounds could be heard in the chest. It was noted that the child held her chin down and resented any attempt to extend the neck.

Mantoux tests, 1 in 10,000 and 1000 , were negative. Bronchoscopy (November 27, 1941) by Mr. Crooks, showed the first part of the trachea

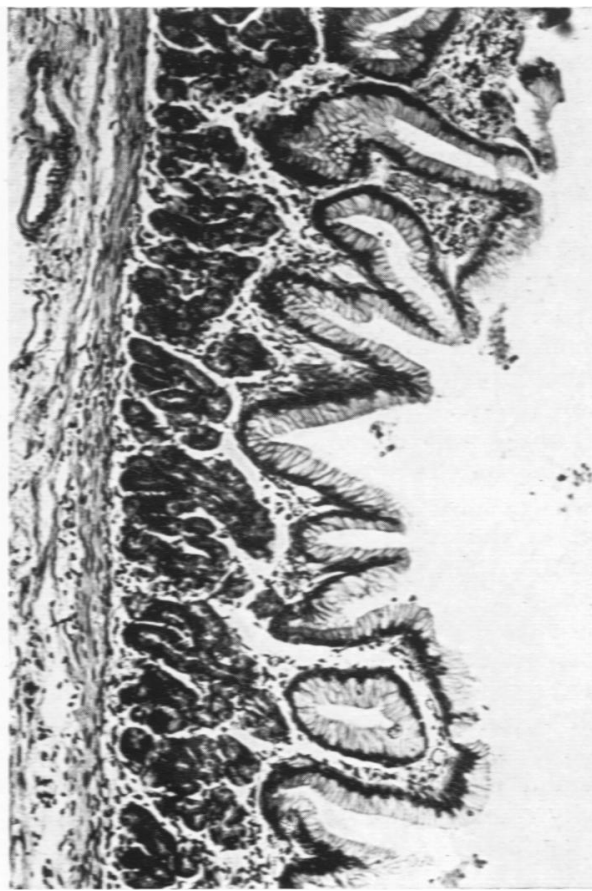

FIG. 6 and 7.-Case 1. Microphotographs of cyst wall showing gastric type of mucous membrane and three layers of plain muscle. 

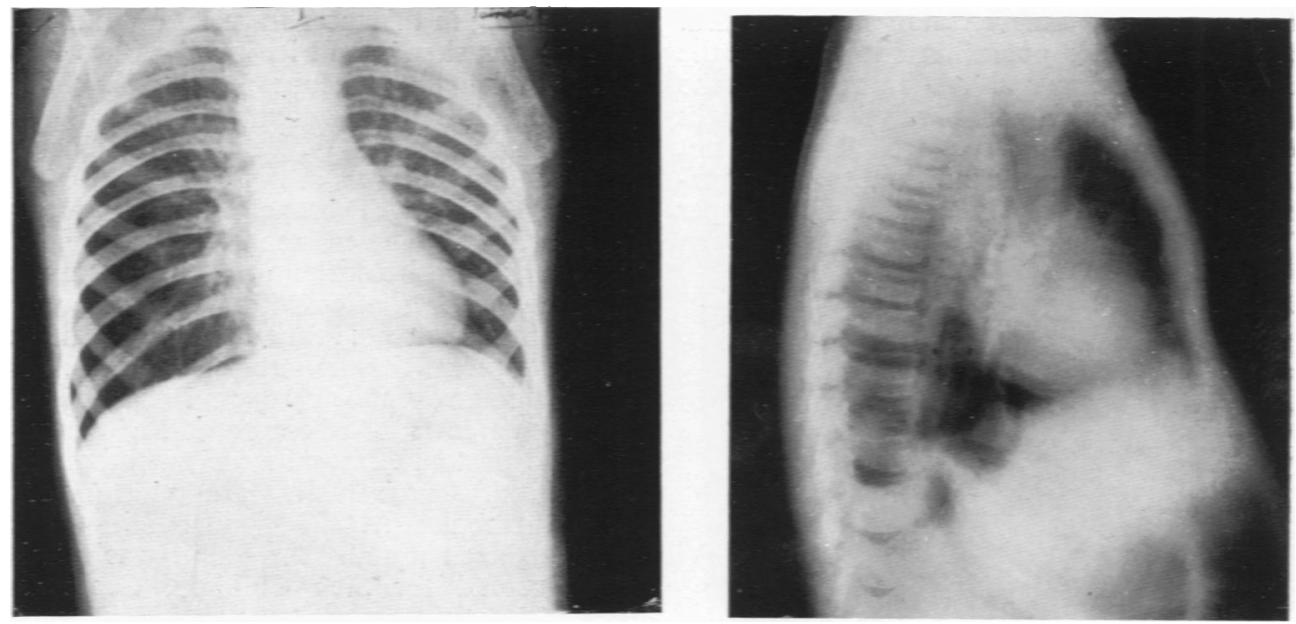

was normal; but the second part, just above the bifurcation, narrowed down to a transverse slit. This appeared to be due to a diffuse swelling of the posterior wall which was patchy and red. Some deficiency of the cartilage rings was suspected at this level. The right and left bronchi were difficult to find but appeared normal. Oesophagoscopy, November 29, 1941 (Mr. Crooks), showed nothing abnormal except some deviation of the oesophagus to the right. An $x$-ray with 'opaque swallow' showed no evidence of obstruction. In a lateral $x$-ray and screening of the chest the trachea was seen to be displaced markedly forward and compressed, curving down over a well-defined mass of uniform opacity, while the oesophagus was displaced backwards and to the right. Perpendicularly the mass lay over the second to sixth dorsal vertebrae. No lesion of cervical or dorsal vertebrae was visible. A provisional diagnosis of postmediastinal tumour, possibly ganglioneuroma, was made, and it was decided that the chest should be explored.

December 16, 1941. Operation by R.S.P. Anaesthetic: intra-tracheal gas, oxygen, ether by Miss Byrde. The chest was opened at the posterior end of the fourth space, a short piece of the posterior end of the fourth rib being resected. The pleura was stripped off the apex of the chest so that the mediastinum could be approached extrapleurally; this was only partly successful and the pleura was torn close to the azygos vein. The oesophagus was identified in the superior mediastinum, being displaced to the right. It appeared larger than normal and inflated with each inspiration. At first no tumour could be found, but after separation of some areolar tissue in front of the oesophagus a smooth soft swelling was exposed to the left of it and behind the trachea. The exposed part had a bluish colour. The azygos vein was then divided in order to increase the exposure, but shortly after this the child's respirations failed, and in spite of artificial respiration the heart also stopped beating and would not respond to massage.

POST-MORTEM EXAMINATION showed both lungs

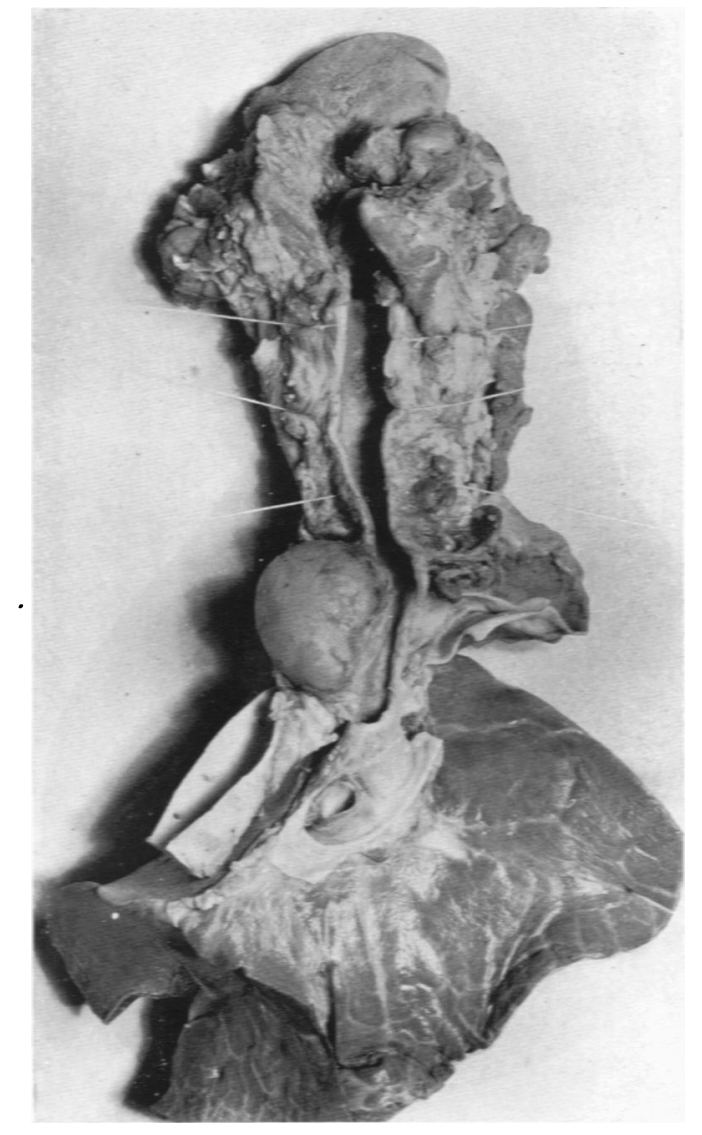

FIG. 10.-Case 2. Post-mortem specimen showing cyst and its relations exposed from behind, the right bronchus has been cut across close to trachea and removed.

were almost completely collapsed. The pleura cavities were not examined for pneumothorax and it was not discovered whether the left pleura had 

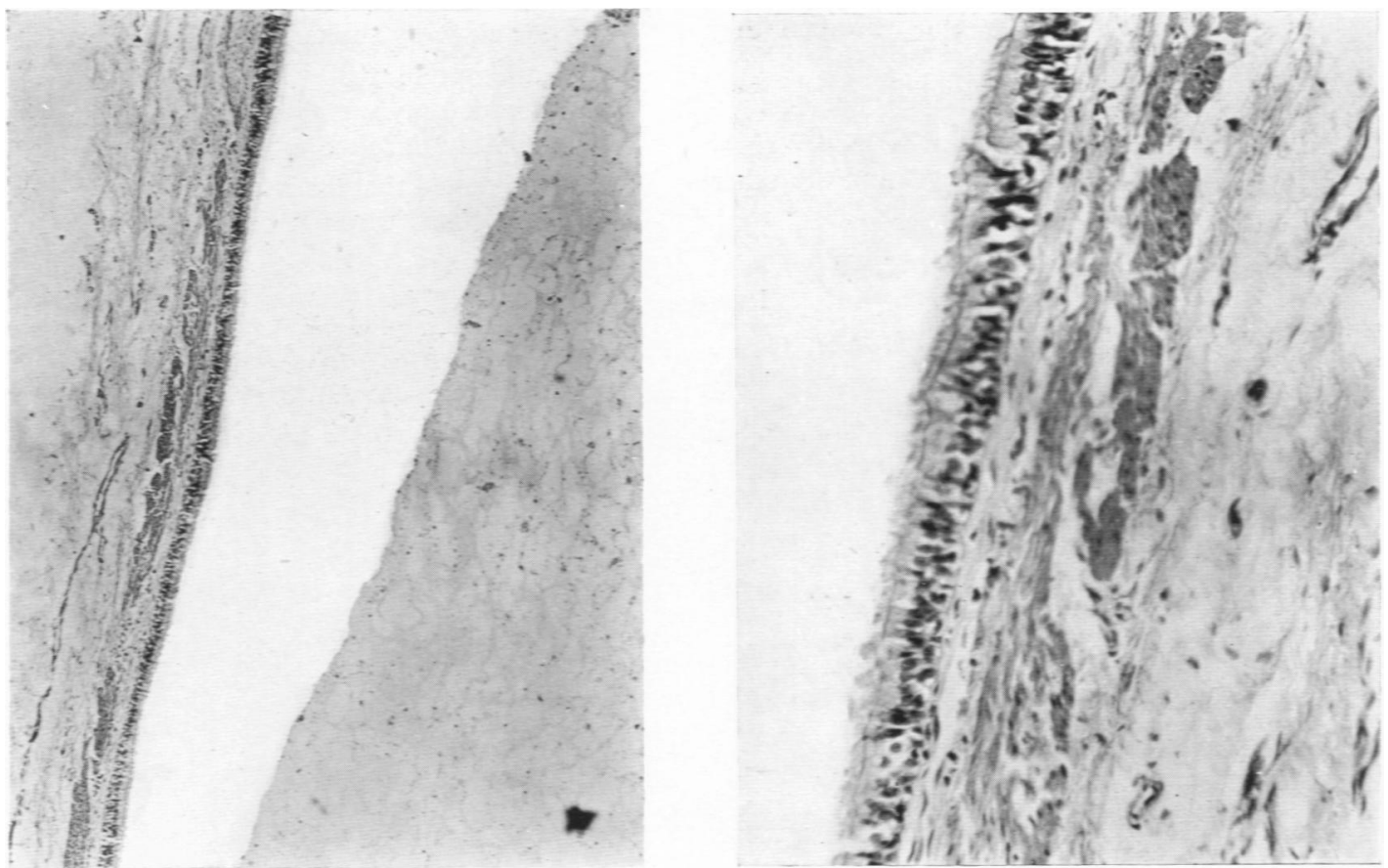

Fig. 11 and 12.-Case 2. Microphotographs of cyst showing epithelium of bronchial type and a single layer of plain muscle.

been torn during operation (this had been suspected as a possible cause of the child's collapse). Arising from the posterior aspect of the left bronchus at its origin was a cystic swelling, $3 \mathrm{~cm}$. in diameter, which was globular in shape. There was a thin covering of mucous membrane over the cyst where it bulged into the trachea and left bronchus, and it looked as if very little violence would have ruptured it into the bronchus. There was some hypertrophy of the left ventricle and it was thought that the cyst might also have pressed on the arch of the aorta. Section of the wall of the cyst showed respiratory epithelium with a thin layer of plain muscle external to it.

\section{Comment}

These two cysts, although probably of similar origin, presented strikingly different clinical pictures and therapeutic problems. In neither case was a pre-operative diagnosis made except in so far as a benign condition amenable to surgery was suspected. The first cyst was large and produced symptoms by occupying space in the chest. There may have been some compression of the right bronchus, but it was not demonstrated and the radiographs suggested that there was some air-containing lung in front. At operation no intimate connexion with other structures in the chest was found and removal in one stage was not technically difficult. The rapid re-expansion of the lung was gratifying, for in such a case if the bronchus were blocked bronchiectasis might occur. It is worth noting that at first the cyst was regarded as a malignant neoplasm and the prognosis hopeless, a mistake which should not occur if the rarer benign conditions such as cysts and nerve tumours are kept in mind. In the second case the cyst was relatively small, but because of its situation and intimate relation to the large air passages, its effects were more serious. In spite of bronchoscopy it was not appreciated that the cyst was in the wall of the left bronchus nor was there any pre-operative suspicion of the collapsed state of the left lung found at post-mortem. This was assumed to be due to bronchial ccelusion although, unfortunately, pneumothorax was not excluded. The problem of how to treat such a cyst is not easy. Exploration was undertaken in the hope of removing it completely, but post-mortem examination showed that this would have been difficult and would probably have necessitated opening the left bronchus. Such a difficulty might have been anticipated if more notice had been taken of the suspected deficiency of the tracheal rings. It is worth considering whether such a cyst should be dealt with through the bronchus. The removal of a portion of the cyst with punch forceps or diathermy should be possible although attended by a risk of infection. No record of successful treatment of a cyst of this type has been found, although several other large right-sided cysts have been successfully removed in stages.

\section{Summary}

1. Cysts of bronchial or intestinal structure or both occur in the posterior mediastinum. 
2. They differ from dermoids in their posterior position and the early age at which they cause symptoms.

3. Symptoms may be due to a large space-occupying lesion or to bronchial obstruction.

4. Two cases, one of a gastric cyst and the other of a bronchial cyst, are described.
5. The gastric cyst was successfully removed in one stage by thoracotomy.

6. An attempt to remove the bronchial cyst by thoracotomy was fatal and the method was probably unsuitable.

7. It is suggested that the latter type of cyst should be attacked through the bronchus.

\section{REFERENCES}

Guillery, H. (1937). Z Zbl.allg. Path. path. Anat., 69,49. Nicholls, M. F. (1940). Brit. J. Surg., 29, 137.

Keibel, F., and Mall, F. P. (1912). Manual of human embryology, Phila. and Lond., 2 ,

Paterson, D., and Pilcher, R. S. (1941). Loc. cit., 608.

Mixter, C. G., and Clifford, S. H. (1929). Ann. Surg., 90, Smith, R. E. (1930). Guy's Hosp. Rep., 80, 466. 714. 